\title{
Was ist Verkehr?
}

Die wichtigste Frage, die wir uns zunächst stellen müssen ist, die nach dem Verkehr selbst. Was verstehen wir unter dem allgemeinen Begriff „Verkehr"?

Unter Verkehr können wir zunächst jede Form der Fortbewegung zwischen mehreren Punkten in unserem Alltagsleben verstehen. Dies ist erst einmal unabhängig davon wie wir uns fortbewegen. Um nicht zu weit in die Ferne zu schweifen, wollen wir den Begriff unterteilen und etwas enger fassen.

Wir unterscheiden zunächst zwischen zwei großen Typen von Verkehr. Beide treffen wir in unserem Alltag häufig an. Da haben wir zum einen die Gruppe des öffentlichen Verkehrs. Hierzu zählen wir all jene Verkehrsmittel, die nicht Einzelpersonen gehören, sondern einer Gruppe von Personen zugeordnet sind wie etwa Züge, Busse, Trambahnen etc. Demgegenüber steht der sogenannte Individualverkehr: PKW, LKW, Motorräder und auch Fahrräder. Diese besitzen wir also privat und nutzen sie in der Regel nicht gemeinschaftlich.

Insbesondere letzterer bereitet uns sowohl im Alltag als auch in der Planung am meisten Kopfzerbrechen. Während der öffentliche Verkehr zumeist vorgegebenen Strukturen folgt - ein Zug etwa seinen Schienen und ein Bus seiner Fahrroute sind die Bewegungen des Individualverkehrs auf den ersten Blick chaotisch und willkürlich für einen außenstehenden Beobachter. Wie soll man dies nur in einer Berechnung erfassen können?

Wir werden im Folgenden sehen, dass es durchaus möglich ist, Ordnung in das Chaos zu bringen und sinnvolle Simulationen und Berechnungen durchführen zu können. Nur auf den ersten Blick sind die Bewegungen der einzelnen Verkehrsteilnehmer unvorhersehbar. Auf den zweiten erkennen wir immer wiederkehrende Strukturen und Muster, die uns helfen werden das Gesamtkonzept Verkehr besser zu erfassen. 